Reprod. Nutr. Dévelop., 1982, 22 (1 B), 235-241.

\title{
Distribution et métabolisme des sites récepteurs cholinergiques pendant la synaptogenèse
}

\author{
J. P. BOURGEOIS, Madeleine TOUTANT
}

Institut Pasteur, Département de Biologie moléculaire, 25, rue du Docteur-Roux, 75724 Paris Cedex 15.

Summary. Distribution and metabolism of cholinergic receptor sites during synaptogenesis.

The effects of a reduction or an increase in muscular activity during embryonic life were investigated in the posterior latissimus dorsi muscle of the chick embryo. The biochemical differentiation, number and localization of the developing neuromuscular junctions were studied after curarization or chronic medullar stimulation. The degradation of junctional as well as extrajunctional PLD cholinergic receptor sites was constant labout $28 \mathrm{~h}$ ) up to day 10 after hatching, and therefore the disappearance of extrajunctional ACh- $\mathrm{R}$ at the end of embryonic life was due to a shut-off of their synthesis. Curarization did not modify the focal innervation of PLD fibres, whereas both the density of extrajunctional $A C h-R$ and neurites per fibre increased. On the other hand, chronic stimulation led to the formation of supernumerary ACh-R clusters of PLD fibres with a parallel increase in the total number of spots of acetylcholinesterase activity per muscle.

\section{Introduction.}

La jonction neuro-musculaire constitue un système modèle de contact synaptique dans l'analyse des mécanismes de différenciation des connexions entre deux ensembles de cellules excitables. Les sites récepteurs physiologiques de l'acétylcholine, localisés dans la membrane plasmique postsynaptique de la jonction neuro-musculaire, constituent l'un des éléments essentiels dans la microphysiologie de la transmission chimique de l'influx nerveux dans cette jonction. La liaison des $\alpha$-neurotoxines sur les récepteurs cholinergiques (nicotoniques) est très spécifique et très faiblement réversible. Elles permettent de décrire le métabolisme et l'évolution de la distribution topologique de ces sites récepteurs sur la surface cellulaire des fibres musculaires lors de la synaptogenèse (Changeux, 1979, 1981). L'embryon de poulet offre la possibilité technique d'étudier et de tester expérimentalement, in situ, certains mécanismes de cette synaptogénèse. Deux muscles striés squelettiques de phénotypes très différents 
ont été étudiés. L'ALD (anterior latissimus dorsi), est un muscle lent à innervation multiple distribuée, dont les unités motrices fonctionnent en permanence à environ $0,5 \mathrm{~Hz}$ et le PLD (posterior lattissimus dorsi) est un muscle rapide à innervation focale unique, dont les unités motrices, peu actives, fonctionnent par de rares bouffées de $8 \mathrm{~Hz}$ (Vrbova et al., 1978).

\section{Matériel et méthodes.}

Le marquage autoradiographique des récepteurs cholinergiques musculaires par $\left.\right|^{\prime 125} \mid-\alpha$-BuTX, ainsi que la mesure de leur demi-vie sont réalisés suivant les techniques décrites dans Betz et al. (1980). La paralysie chronique des embryons de poulet est obtenue par des injections répétées de Flaxédil (agent curarisant, May et Baker, $10 \mathrm{mg}$ dans $100 \mu \mathrm{l} \mathrm{NaCl}$ à 0,9 p. 100) les $4,7,10$ et $13^{e}$ jours de l'incubation (Bourgeois et al., 1978). La stimulation médullaire chronique est réalisée, chez l'embryon de poulet, à partir du $10^{\circ}$ jour in ovo, par l'intermédiaire de 2 électrodes de platine, préalablement implantées autour de la moelle épinière de part et d'autre de la zone d'innervation motrice du PLD, le 7 e jour de l'incubation (Toutant et al., 1980).

\section{Résultats. Discussion.}

1. Distribution et métabolisme des sites récepteurs cholinergiques extrasynaptiques.

Sur des frottis de fibres musculaires embryonnaires isolées du muscle PLD, la densité absolue des sites récepteurs cholinergiques (nicotiniques) augmente du $4^{e}$ au $11^{\mathrm{e}}$ jour jusqu'à un maximum de 220 sites par $\mu^{2}$, puis diminue pour s'annuler au 19e jour in ovo (Betz et al., 1977, 1980).

Deux mécanismes différents peuvent rendre compte de cette disparition des sites récepteurs extrasynaptiques : (1) soit un arrêt de leur synthèse ; (2) soit une accélération de leur vitesse de dégradation. Des prélèvements de muscle PLD ou pectoraux à des temps successifs après des injections intraembryonnaires $d^{\prime} \alpha-$ Bungarotoxine iodée, ont montré que la vitesse de dégradation de la protéine réceptrice cholinergique ne varie pas significativement entre le $8^{\mathrm{e}}$ et le $18^{\mathrm{e}}$ jour in ovo, avec une demi-vie d'environ 28 h (Betz et al., 1980).

La disparition des sites récepteurs extrasynaptiques apparaît donc liée à un arrêt de leur synthèse. Cet arrêt de la synthèse est contrôlé par l'activité motrice puisque une paralysie chronique induite par un agent curarisant fait doubler le nombre total de sites récepteurs sans modifier leur demi-vie (Bourgeois et al., 1978). Ce mécanisme est commun aux fibres musculaires chez les animaux adultes après dénervation et aux myotubes cultivés in vitro (voir revues dans Fambrough, 1979 et Thesleff et Sellin, 1980). Le couplage entre l'activité physiologique des fibres musculaires et la régulation de la synthèse de la protéine réceptrice cholinergique met en jeu le $\mathrm{Ca}^{+}{ }^{+}$et les nucléotides cycliques comme le GMP cyclique (Nestler et al., 1978 ; Betz et Changeux, 1979). 


\section{Formation et maturation des jonctions neuromusculaires.}

Des amas de sites récepteurs cholinergiques, révélés par les $\alpha$-neurotoxines radioactives, sont présents dès le $4^{e}$ jour in ovo dans le blastème pré-musculaire du muscle PLD. Leur nombre total par muscle n'augmente réellement que après le $11^{\mathrm{e}}$ jour in ovo, de manière régulière et asynchrone (Betz et al., 1980). Un seul amas de sites récepteurs, correspondant à la plaque motrice, est présent sur chaque fibre musculaire PLD embryonnaire. Cette zone sous-synaptique, qui ne représente que le $1 / 10000^{\circ}$ de la surface totale de la fibre musculaire, a une densité de sites récepteurs de 10 à 100 fois supérieure à celle trouvée dans les zones extrasynaptiques (Burden, 1977 a). L'agrégation des sites récepteurs ne coïncide pas nécessairement avec leur stabilisation métabolique, puisque leur demi-vie dans les amas de sites est identique $(\sim 30 \mathrm{~h})$ à celle des sites récepteurs extrasynaptiques (Burden, 1977 b). Chez le poulet, la stabilisation métabolique des sites récepteurs sous-synaptiques intervient seulement un mois après l'éclosion (Betz et al., 1980), et pourrait être associée à des réactions de phosphorylation (Saitoh et Changeux, 1981). Chez le rat nouveau-né, cette stabilisation métabolique est très rapide (Michler et Sakman, 1980). La paralysie des muscles PLD induite par des injections répétées de Flaxedil, prévient la localisation sous-synaptique de l'acétylcholinestérase détectable histochimiquement, mais ne prévient pas la localisation des sites récepteurs cholinergiques révélés par l' $\alpha$-Bungarotoxine (Bourgeois et al., 1978 ; Betz et al., 1980).

L'activité motrice des fibres musculaires embryonnaires semble donc contrôler d'une part la synthèse des sites récepteurs cholinergiques extrasynaptiques et la localisation des sites acétylcholinestérasiques sous-synaptiques; mais elle ne contrôle pas directement la localisation sous-synaptique des sites récepteurs ni l'activité acétylcholinestérasique dans le muscle.

Cette différence de régulation métabolique pourrait être liée au fait que ces deux protéines suivent des voies intracellulaires différentes (Smilowitz, 1980) mais ce fait est controversé (Rotundo et Fambrough, 1980).

La formation de contacts synaptiques primitifs, comportant des « Gap junctions 》 entre neurites et myotubes (Peng et al., 1980) pourrait précéder l'étape d'agrégation des sites récepteurs cholinergiques sous la terminaison nerveuse motrice. Cette agrégation se fait soit par diffusion latérale des sites récepteurs dans le plan de la membrane plasmique (revue dans Fambrough, 1979) soit par un processus d'exo-endocytose locale (Peng et al., 1980). Elle précède la localisation de l'acétylcholinestérase (Vrbova et al., 1978 ; Rubin et al., 1980 ; J. Koenig, communication personnelle). La maturation de la plaque motrice se manifeste également par une transformation de ses propriétés électrophysiologiques. In vivo, chez le rat nouveau-né, pendant la première quinzaine de jours postnatale, a lieu une conversion de l'ionophore du type "embryonnaire » à ouverture lente, vers le type " adulte ", sous-synaptique, à ouverture rapide. Cette modification métabolique ou structurelle de l'ionophore sous la terminaison nerveuse motrice fonctionnelle est postérieure à la stabilisation métabolique du site récepteur et relève probablement d'un mécanisme différent, pour l'instant inconnu (Michler et 
Sakman, 1980 ; Schuetze, 1980). A cette maturation fonctionnelle de la plaque motrice est associée également une maturation morphologique : $d$ 'abord un agrandissement spectaculaire de sa taille qui, par exemple dans le muscle PLD du poulet, passe d'une dizaine de microns carrés chez l'embryon, à près de 1000 microns carrés un mois après l'éclosion (Burden, 1977 a ; Betz et al., 1980 ; Toutant et al., 1980). Cette croissance se ferait à densité constante des sites récepteurs (Burden, 1977 a). Simultanément la structure de la plaque motrice se modifie : elle passe progressivement de la forme lenticulaire chez le jeune poussin (Burden, 1977 a ; Bourgeois et al., 1978 ; Betz et al., 1980) à la forme " en plaque " chez l'adulte (Atsumi, 1977; Koenig, 1970 ; voir aussi Michler et Sakman, 1980 pour le rat). Cette maturation complexe de la plaque motrice se traduit par une évolution continue des propriétés physiologiques du contact synaptique, lui donnant une capacité de réponse de plus en plus efficace et rapide à l'arrivée des influx nerveux dans la terminaison nerveuse motrice.

\section{Distribution topologique des plaques motrices.}

Les conditions expérimentales, réalisées soit chez l'animal adulte, lors d'expériences de réinnervation (Lomo, 1980), soit dans les co-cultures in vitro (Giacobini, 1980 ; J. Koenig, communication personnelle), ne sont pas rigoureusement identiques à celles rencontrées chez l'embryon lors de la mise en place du réseau neuronal. Pour ces raisons, nous avons choisi d'étudier cette régulation de la synaptogenèse in situ sur l'embryon de poulet.

Dans le modèle de régulation de la distribution topologique des contacts neuromusculaires proposé par Vrbova et al. (1978), le nombre et la distribution des plaques motrices sur une fibre musculaire dépendent de la capacité de chaque terminaison nerveuse à dépolariser une certaine étendue de la fibre musculaire. L'étendue de fibre musculaire ainsi contrôlée par une terminaison motrice constitue une zone réfractaire à la formation de toute autre jonction. La prévision de ce modèle est qu'une réduction de l'activité synaptique augmente le nombre de contacts synaptiques sur une fibre musculaire. Sa vérification expérimentale, consiste à réduire, par une curarisation chronique, la quantité de neurotransmetteur reçue au niveau de la membrane plasmique postsynaptique. Des injections répétées de Flaxedil, du $4^{\mathrm{e}}$ au $13^{\mathrm{e}}$ jour in ovo ne modifient cependant ni le nombre, ni la distribution topologique des amas de sites récepteurs cholinergiques sur les fibres musculaires du muscle PLD au $15^{\mathrm{e}}$ jour in ovo. Le nombre de sites récepteurs extrasynaptiques augmente (Bourgeois et al., 1978), de même que le nombre total de terminaisons nerveuses motrices dans le muscle PLD (Bourgeois et Toutant, en préparation). Ces observations montrent que l'hypersensibilité cholinergique extrasynaptique, ainsi que la présence d'un grand nombre de neurites, ne sont pas suffisantes, per se, pour induire l'apparition de plaques motrices surnuméraires sur les fibres musculaires PLD paralysées.

Dans le modèle de la " stabilisation sélective " proposé par Changeux et Danchin, 1976, la stabilisation d'une jonction neuro-musculaire dépend ici aussi de son état fonctionnel ; mais c'est l'information transportée par les fibres nerveuses afférentes, sous la forme d'influx nerveux, qui est transformée en information de 
position topologique (Changeux et Danchin, 1974, 1976). La prévision de ce modèle de la " stabilisation sélective " est que l'augmentation du nombre de contacts synaptiques le long d'une fibre musculaire est liée à une augmentation de la fréquence de fonctionnement du système motoneurone-fibre musculaire, et plus précisément de leurs contacts synaptiques. Une vérification expérimentale de ce modèle a consisté à imposer une stimulation électrique chronique à la moelle épinière d'embryons de poulet pendant la période de formation des contacts synaptiques. Par l'intermédiaire d'électrodes implantées autour du tube neural au 7 e jour in ovo, des chocs électriques (10 volts, $5 \mathrm{msec})$ sont délivrés de manière chronique, du $10^{\circ}$ au $15^{\circ}$ ou $16^{\circ}$ jour in ovo, avec une fréquence de décharge de $0,5 \mathrm{~Hz}$ de type ALD (Vrbova et al., 1978). Cette augmentation de l'activité physiologique imposée au muscle PLD a pour résultat un doublement du nombre total d'amas de sites récepteurs cholinergiques et d'amas de sites acétylcholinestérasiques par muscle PLD (tabl. 1 ; Toutant et al., 1980, 1981) sans que le nombre total de fibres musculaires ni celui des axones moteurs ne changent (Bourgeois et Toutant, en préparation). L'apparition d'amas surnuméraires de sites récepteurs (Toutant et al., 1980) ou de sites acétylcholinestérasiques détectés histochimiquement (Toutant et al., 1979) distribués le long des fibres musculaires PLD stimulées, prédite par le modèle de Changeux peut cependant avoir plusieurs explications différentes (Toutant, 1979).

\section{TABLEAU 1}

Effets de la stimulation médullaire chronique sur le nombre total d'amas de récepteurs (colonne 1) et de sites acéty/cholinestérasiques (colonne 2) par muscle PLD aux stades embryonnaires 40 (14 jours) et 41 (15 jours d'incubation). Les valeurs sont des moyennes \pm erreur standard.

\begin{tabular}{|c|c|c|c|}
\hline Embryons & $\begin{array}{l}\text { Nombre total d'amas } \\
\text { de sites récepteurs } \\
\text { par muscle PLD }\end{array}$ & $\begin{array}{c}\text { Nombre total } \\
\text { de fibres musculaires } \\
\text { par muscle PLD }\end{array}$ & $\begin{array}{l}\text { Nombre total d'amas } \\
\text { de sites acétylcho- } \\
\text { linestérasiques } \\
\text { par muscle PLD }\end{array}$ \\
\hline Témoins & $\begin{array}{c}3831 \pm 145 \\
(n=3)\end{array}$ & $\begin{array}{c}4308 \pm 636 \\
(n=4)\end{array}$ & $\begin{array}{c}5581 \pm 1028 \\
(n=4) \\
\text { stade } 41\end{array}$ \\
\hline $\begin{array}{l}\text { Stimulation } \\
\text { médullaire } \\
\text { chronique }\end{array}$ & $\begin{array}{c}6923 \pm 572 \\
(n=4)\end{array}$ & $\begin{array}{c}4633 \pm 506 \\
(n=4)\end{array}$ & $\begin{array}{c}12660 \pm 1138 \\
(n=4) \\
\text { stade 41) }\end{array}$ \\
\hline
\end{tabular}

L'ensemble de ces résultats montre que l'activité physiologique du système motoneurone-fibre musculaire joue un rôle important, sinon exclusif, dans l'évolution de la distribution des différentes protéines cholinergiques ainsi que dans la mise en place des jonctions neuromusculaires. 


\section{Références}

ATSUM। S., 1977. Development of neuromuscular junctions of fast and slow muscles in the chick embryo : a light and electron microscopic study. J. Neurocytol., 6, 691-709.

BETZ H., BOURGEOIS J.-P., CHANGEUX J. -P., 1977. Evidence for degradation of the acetylcholine (nicotinic) receptor in skeletal muscle during the development of the chick embryo. FEBS Lett., 77, 219-224.

BETZ H., BOURGEOIS J.-P., CHANGEUX J.-P., 1980. Evolution of cholinergic proteins in developing slow and fast skeletal muscles in chick embryo. J. Physiol. (London), 302, 197-218.

BETZ H., CHANGEUX J.-P., 1979. Regulation of muscle acetylcholine receptor synthesis in vitro by derivatives of cyclic nucleotides. Nature, 278, 749-752.

BOURGEOIS J.-P., BETZ H., CHANGEUX J.-P., 1978. Effets de la paralysie chronique de l'embryon de poulet par le flaxedil sur le développement de la jonction neuromusculaire. $C$. $R$. Acad. Sci. Paris, sér. D, 286, 773-776.

BURDEN S., 1977 a. Development of the neuromuscular junction in the chick embryo : the number, distribution and stability of acetylcholine receptors. Dev. Biol., 57, 317-329.

BURDEN S., 1977 b. Acetylcholine receptors at the neuromuscular junction : developmental change in turnover. Dev. Biol., 61, 79-85.

CHANGEUX J.-P., 1979. Molecular interactions in adult and developing neuromuscular junction. The Neurosciences Fourth Study Program. MIT Press, Cambridge, Ma, 749-778.

CHANGEUX J.-P., 1981. The Acetylcholine Receptor: an allosteric membrane protein. Harvey Lectures (sous presse).

CHANGEUX J.-P., DANCHIN A., 1974. Apprendre par stabilisation sélective de synapses en cours de développement, 320-357. In L'Unité de I'Homme, Le Seuil, Paris.

CHANGEUX J.-P., DANCHIN A., 1976. Selective stabilisation of developing synapses as a mechanism for the specification of neuronal networks. Nature, 264, 705-713.

FAMBROUGH D., 1979. Control of acetylcholine receptors in skeletal muscle. Physiol. Rev., 59, 165227.

GIACOBINI E., 1980. Discrepencies and differences between nerve cells growing in vitro and in situ : a discussion, 187-210. In Tissue culture in neurobiology, Raven Press, New York.

KOENIG J., 1970. Contribution à l'étude de la morphologie des plaques motrices des grands dorsaux antérieur et postérieur du poulet après innervation croisée. Arch. Anat. microsc. Morph. exp., 59, 403-425.

LOMO T., 1980. What controls the development of neuromuscular junctions. Trends Neurosci, 3, 126-129.

MICHLER A., SAKMAN B., 1980. Receptor stability and channel conversion in the subsynaptic membrane of the developing mammalian neuromuscular junction. Dev. Biol., 79, 125-141.

NESTLER E. J., BEAM K. G., GREENGARD P., 1978. Nicotinic cholinergic stimulation increases cyclic GMP levels in vertebrate skeletal muscle. Nature, 275, 4b1-453.

PENG H.B., NAKAJIMA Y., BRIDGMAN P.C., 1980. Development of the post-synaptic membrane in Xenopus neuromuscular cultures observed by freeze fracture and thin section electron microscopy. Brain Res., 196, 11-31.

ROTUNDO R.L., FAMBROUGH D., 1980. Secretion of acetylcholinesterase : relation to acetylcholine receptor metabolism. Cell, 22, 595-602.

RUBIN L. L., SCHUETZE M., WEILL C. L., FISCHBACH G. D., 1980. Regulation of acetylcholinesterase appearance at neuromuscular junctions in vitro. Nature, 283, 264-267.

SAITOH T., CHANGEUX J.-P., 1981. Change in the state of phosphorylation of the acetylcholine receptor during maturation of the electromotor synapse in Torpedo marmorata electric organ. Proc. nat. Acad. Sci. USA (sous presse).

SCHUETZE S. M., 1980. The mean channel open time in chick muscle is not decreased following innervation. J. Physiol. (London), 303, 111-124.

SMILOWITZ H., 1980. Routes of intracellular transport of acetylcholine receptor and esterase are distinct. Cell, 19, 237-244. 
THESLEFF S., SELLIN L.C., 1980 . Denervation supersensitivity. Trends Neurosci, 3, 122-126.

TOUTANT M., 1979. Effets de la stimulation médullaire chronique sur la localisation des récepteurs cholinergiques musculaires du grand dorsal postérieur chez l'embryon de poulet. Th. Doct. $3^{e}$ cycle, Nantes.

TOUTANT J.P., TOUTANT M., RENAUD D., LE DOUARIN G., 1979. Enzymatic differentiation of muscle fibre type in embryonic latissimus dorsii of the chick: effects of spinal cord stimulation. Cell Diff., 8, 375-382.

TOUTANT M., BOURGEOIS J.P., TOUTANT J.P., RENAUD D., LE DOUARIN G., CHANGEUX J.-P., 1980. Chronic stimulation of the spinal cord in developing chick embryo causes the differentiation of multiple clusters of acetylcholine receptor in the posterior latissimus dorsi muscle. Dev. Biol., 76, 384-395.

TOUTANT M., TOUTANT J.P., RENAUD D., LE DOUARIN G., CHANGEUX J.-P., 1981. Effet de la stimulation médullaire chronique sur le nombre total de sites d'activité acétylcholinestérasique du muscle posterior latissimus dorsi de l'embryon de poulet. C. R. Acad. Sci. Paris, sér. III, 292, 771-775.

VRBOVA G., GORDON T., JONES R., 1978. Nerve-muscle interaction. Chapman \& Hall, London. 